\title{
The Sentinel Hospitalization and the Role of Palliative Care
}

\author{
Richard J. Lin, MD, PhD, Ronald D. Adelman, MD², Randi R. Diamond, MD², Arthur T. Evans, MD, MPH
}

${ }^{1}$ Division of Hospital Medicine, Department of Medicine, Weill Cornell Medical College, New York, New York; ${ }^{2}$ Division of Geriatrics and Palliative Medicine, Department of Medicine, Weill Cornell Medical College, New York, New York.

With current healthcare reform and calls for improving care quality and safety, there is renewed emphasis on highvalue care. Moreover, given the significant healthcare resource utilization for patients with chronically progressive illnesses or for patients at the end of life, innovative and efficient care delivery models are urgently needed. We propose here the concept of a sentinel hospitalization, defined as a transitional point in the patient's disease course that heralds a need to reassess prognosis, patient understanding, treatment options and intensities, and goals of care. Hospitalists are well positioned to recognize a patient's sentinel hospitalization and use it as an opportunity for active integration of palliative care that provides high-quality and cost-saving care through its patient- and family-oriented approach, its interdisciplinary nature, and its focus on symptom control and care coordination. Journal of Hospital Medicine 2014;9:320-323. (C) 2014 Society of Hospital Medicine
As hospitalists now care for expanding numbers of America's aging patients, many of whom have chronic, debilitating illnesses or are near the end of life, there is a burgeoning need for innovative approaches to optimize quality of care and control costs, especially in the last year of life. ${ }^{1,2}$ In the inaugural issue of the Journal of Hospital Medicine, an overview of how hospitalists and palliative care specialists can work hand-in-hand to care for these seriously ill, hospitalized patients was presented. ${ }^{3,4}$ This perspective highlighted a symbiotic and mutually beneficial relationship between the 2 specialties based on their shared values, missions, and complementary strengths. ${ }^{3,4}$ Since then, a number of collaborative ventures offering palliative care for seriously ill, hospitalized patients have been developed and examined in a variety of settings. ${ }^{4-6}$

A key collaborative undertaking for hospitalists and palliative care specialists is the appreciation of the unique trajectory of each chronic illness toward the end of life. For example, patients with cancer or neurodegenerative disease tend to have relatively stable functional status until the final months of rapid deterioration. On the other hand, the courses of patients with chronic obstructive pulmonary disease (COPD), congestive heart failure $(\mathrm{CHF})$, end-stage renal disease, and human immunodeficiency virus/acquired immunodeficiency syndrome tend to be punctuated by episodes of acute exacerbation with often nearly complete return to previous status. Finally, dementia

\footnotetext{
*Address for correspondence and reprint requests: Richard J. Lin, MD, Department of Medicine, Weill Cornell Medical College, 525 East 68th Street, Box 130, New York, NY 10065; Telephone: 212-746-9832; Fax: 212-746-4734; E-mail: ril9016@med.cornell.edu

Additional Supporting Information may be found in the online version of this article.

Received: August 20, 2013; Revised: December 23, 2013; Accepted: January 8, 2014

2014 Society of Hospital Medicine DOI 10.1002/jhm.2160

Published online in Wiley Online Library (Wileyonlinelibrary.com).
}

usually follows a slow course of gradual decline leading to death. ${ }^{7}$ Ideally, active management of symptoms and discussion of prognosis and goals of care should happen in the early stages of these chronic illnesses, yet most often they are left until an acute hospitalization late in the disease course. The following case illustrates the point.

\section{CASE 1}

Mrs. $\mathrm{M}$ is an 89-year-old woman with Alzheimer's dementia diagnosed 7 years ago who has been cared for at home by family members. She is admitted to the hospital for urinary tract infection and volume depletion. She is bedbound, cachectic, and has a stage III decubitus ulcer. Her daughter describes a 6-month history of feeding problems, 20-lb weight loss, and 2 recent hospitalizations for aspiration pneumonia. She improves somewhat with hydration and intravenous antibiotics, and the physical therapist recommends rehabilitation. Mrs. M does not have decision-making capacity, and her long-time family physician has not inquired about care preferences or goals. The hospitalist team meets with family members to discuss the trajectory and prognosis of advanced dementia, and recommends against artificial nutrition and hydration, and for initiation of palliative care service at a skilled nursing facility.

In this example, the hospitalist team recognizes the advent of frequent infections and diminished oral intake in advanced dementia as signals of increased morbidity and mortality warranting palliative care intervention. ${ }^{8}$ This, we suggest, represents a sentinel hospitalization, a hospitalization in the disease course that heralds a need to reassess prognosis, treatment options and intensity, and goals of care. Hospitalists are well positioned to recognize such transition points in the disease course by considering the patient's recent history of illness, to offer an impartial overview of illness progression, and to optimize patient care 
TABLE 1. Common Criteria Defining a Sentinel Hospitalization

\begin{tabular}{lcc}
\hline Common Criteria & Time Frame & References \\
\hline $\begin{array}{l}\text { "No" to the "surprise" question: Would you } \\
\text { be surprised if the patient died in } 12 \text { months? }\end{array}$ & 1 year & 10,11 \\
Newly diagnosed metastatic solid cancer & Various & 17 \\
Metastatic solid cancer admitted for uncontrolled symptoms & Various & 17 \\
Progressive CKD with consideration for hemodialysis & 1 year & 17,18 \\
GOLD stage IV COPD with frequent exacerbation & Various & 20 \\
NYHAstage IV CHF with frequent exacerbation & $1-2$ years & 21 \\
Advanced dementia with frequent UTI, aspiration & $1-2$ years & 8 \\
PNA, and feeding problem & & \\
Overall prognosis of high mortality using & 30 days-1 year & $12-14$ \\
available indices & & \\
More than 3 admissions in last 6 months & 6 months & 17,19 \\
Prolonged ICU stay (>7 days) & Weeks & 17,19 \\
\hline
\end{tabular}

NOTE: Abbreviations: CHF, congestive heart failure; CKD, chronic kidney disease; COPD, chronic obstructive pulmonary disease; GOLD, Global Initiative for Chronic Obstructive Lung Disease; ICU, intensive care unit; NYHA, New York Heart Association; PNA, pneumonia; UTI, urinary tract infection.

using principles of palliative care. Additionally, hospitalists have advantages of geographic convenience, readily available consultants, systemic support, and a detachment from the longitudinal patient-physician relationship, which may enable more accurate medical prognostication. ${ }^{9}$

There are many ways to identify a sentinel hospitalization. For example, hospitalists can use the "surprise" question, "Would you be surprised if the patient died within 12 months?" on admission for the majority of cancer and dialysis patients. The answer "No" predicts a 3.5 - to 7 -fold increase in 1 -year mortality. ${ }^{10,11}$ In a powerful predictive model for 1-year mortality using readily available clinical, laboratory, and functional characteristics, medical inpatients in the highest quartiles have 1-year mortality exceeding $60 \% .^{12}$ Recently, several more complicated prognostic models have been derived and validated in large cohorts of medical inpatients, which predict shortterm (30-day) and long-term (0.5-1 year) mortality with great accuracy. ${ }^{13,14}$ There are also many diseasespecific prognostic features (eg, diagnosis of metastatic disease with poor performance status or high symptom burden, progression of chronic kidney disease with consideration of hemodialysis, additional stroke in multi-infarct dementia, and frequent exacerbation of severe COPD or severe CHF). ${ }^{15-21}$ Finally, frequent readmissions and prolonged hospital or intensive care unit stay can also be used. ${ }^{17,19}$ These criteria are summarized in Table 1 with time frames.

Once a sentinel hospitalization is identified, hospitalists, with input from the patient's primary care physician and subspecialists, can then develop a comprehensive strategy to evaluate current disease management, to educate patient and family accordingly, and to actively integrate palliative care services as appropriate. The next challenge facing the care team is how to deliver the necessary palliative care since it is unneces- sary and improper to ask for palliative care specialist consultation for every sentinel hospitalization. We believe that the best approach is for hospitalists to be the primary deliverers of basic palliative care in the hospital while consulting palliative care specialists for refractory symptoms and complex scenarios. ${ }^{22}$ According to this generalist-specialist palliative care model, physicians of all specialties should define and master a basic palliative care skill set for their patients. For hospitalists, the relevant skill set includes assessing and treating pain and other symptoms such as dyspnea, nausea and vomiting, and constipation, estimating prognosis, and initiating goals of care discussions. ${ }^{22}$ The following case illustrates this point.

\section{CASE 2}

Ms S, a 21-year-old Hispanic woman with advanced, recurrent head and neck cancer, status post multiple surgeries, chemotherapy, and radiation therapy, is admitted to the hospitalist service for aspiration pneumonia, which responds to antibiotics rapidly. However, her cancer-related somatic and neuropathic pain soon becomes refractory to opioids prescribed by the hospitalist team. She also develops significant dyspnea, xerostomia, depression, anxiety, and existential suffering. With the help of the interdisciplinary palliative care team, her pain is relieved by a patient-controlled analgesia pump and methadone. A palliative care social worker and chaplain visit her and her family daily to address their distress. Eventually, the care team is able to provide a stable medical regimen for symptom control and to use it across the entire care continuum.

In this example, the hospitalist team, with the support of palliative care specialists, provided basic palliative care and longitudinal integration of palliative practices into the patient's overall treatment scheme. Hospitalists, given their scope of practice and sheer volume of patients, are well positioned to rapidly gain competencies in symptom management, empathic communication, and interdisciplinary teamwork. ${ }^{23,24}$ Hospitalists may benefit from innovative and collaborative palliative care education using interactive online modules, case simulation, communication workshops, and observed evaluation and feedback. ${ }^{25}$ Several modes of collaboration between hospital medicine and palliative care have been developed including implementation of palliative care consult triggers on admission, palliative care participation in hospitalist interdisciplinary rounds, and disease specific, integrated management programs. ${ }^{17,26}$ These collaborations are particularly important, as the quality of inpatient care at the end of life is still suboptimal and more appropriate use of palliative care will be beneficial. ${ }^{27}$ Recently, some hospitals have developed specialized inpatient palliative care units, combining intensive palliation with inpatient medical surgical level of care, as well as providing hospice care. Staffed by palliative care specialists or hospitalists, they provide efficient, cost-saving care to 
patients with advanced chronic illness or terminal disease in need of intensive symptom management. ${ }^{28}$ Finally, there is mounting evidence supporting the clinical effectiveness of palliative care in diverse specialties such as oncology, pulmonary and critical care, and nephrology. ${ }^{29}$ For example, in the setting of metastatic non-small cell lung cancer, early initiation of palliative care has been shown to improve symptom control and quality of life, reduce chemotherapy use at the end of life, and interestingly, prolong median survival by almost 3 months. ${ }^{30}$ This has led to a position statement from American Society of Clinical Oncology encouraging early integration of palliative care into standard oncologic care for advanced disease. ${ }^{31}$

Recognizing a sentinel hospitalization allows palliative care to be integrated at transitions of care and carried forward. For patients with chronic debilitating illnesses who are approaching the end of life, appropriate care transitions will ensure that their short- and long-term care matches their goals of care, assure timely clinical follow-ups, and help reduce hospital readmission and healthcare resource utilization. ${ }^{32}$ Importantly, timely and compassionate communication is a key to the success of both hospital medicine and palliative care. Many patients with life-limiting diseases prefer to receive prognostic information and to discuss goals of care. ${ }^{33}$ How this information is integrated and communicated through the care continuum is crucial, especially in the era of duty hour limits and frequent handoffs. The information exchange needs to facilitate active participation of primary care physicians who may not be involved in hospital care. Some of the innovative strategies for communication and transfer of palliative care information, such as prognosis, goals of care, family meeting consensus, and symptom control interventions, include a palliative care checklist in the electronic health record, incorporation of prognostic and family meeting information in the discharge summary, and links to the national Physician Orders for Life-Sustaining Treatment advanced care planning program. ${ }^{34}$ Of note, a pilot program in the United Kingdom adopting an electronic palliative care summary has reduced afterhour emergency room visits and hospital readmissions. ${ }^{35}$ The following case illustrates this point.

\section{CASE 3}

Mrs. K, an 82-year-old Russian-speaking woman with newly diagnosed metastatic pancreatic cancer, is admitted for worsening obstructive jaundice and a second opinion about treatment. A biliary stent is placed and her jaundice slowly improves. The patient and family have requested chemotherapy. However, the oncologist determines that she would only qualify for a phase I trial given her poor performance status. The hospitalist team requests the help of the palliative care consult team to manage her severe pain, depression, and to provide support to the family. After several family meetings, the patient and family choose not to pursue chemotherapy. Given the lack of adequate support at home, she is discharged to a skilled nursing facility for short-term rehabilitation with plans to transition to the in-house hospice program. The hospitalist, palliative care attending physician, and the medical director of the rehabilitation facility have a 3-way phone conference to confirm the plans of care and to ensure a smooth care transition.

In this case, the hospitalist team recognizes that this is a sentinel hospitalization for Mrs. $\mathrm{K}$ that requires extensive palliative care intervention. Often, transitioning to skilled nursing facilities (SNF) is the default pathway for patients needing hospice/palliative care, especially when patients and families are not yet ready to discuss prognosis realistically or to accept hospice, or there is not enough support available at home. A recent large cohort study showed that $30 \%$ of patients in their last 6-month of life had used, and nearly $10 \%$ of such patients had died, under Medicare's posthospitalization SNF benefit. ${ }^{36}$ Although the worsening disease trajectory may not be apparent at hospital discharge, it is more likely that the financial and practical limitation of the Medicare Hospice Benefit accounts for this observation, which includes limited home health aid hours, lack of coverage for room and board, and lower payments to SNFs. ${ }^{36}$ Hospitalists can help address the issue of discharge location for patients needing palliative care. Sometimes this requires extensive communication before and after discharge to help enhance the transition from a rehabilitation facility to hospice/palliative care. Appropriately integrated palliative care at the time of care transitions, in the form of hospice or longitudinal home-based palliative care rather than just routine clinic follow-up, has the potential to reduce 30-day readmission for chronically ill, elderly patients and for patients near the end of life. ${ }^{37,38}$ It is critical that national policy, suitable reimbursement, and financial incentives support this practice. A demonstration project, Better Outcomes by Optimizing Safe Transitions (BOOST), organized by the Society of Hospital Medicine, integrates palliative care evaluation into a comprehensive discharge assessment tool. This intervention has been shown to reduce readmissions to acute care hospitals. ${ }^{39}$

In this article, we define a sentinel hospitalization and suggest that its recognition provides an important opportunity for hospitalists to actively integrate palliative care into patients' chronic disease management programs, with inputs from patients, their families, their primary physicians and subspecialists, as well as palliative care specialists. We also recognize that within nonsentinel hospitalizations, there are important opportunities to discuss prognosis, goals of care, and advanced care planning. This approach allows the "fresh eyes" of hospitalists to assess the patient's current health status and prognosis, to communicate these relevant clinical issues with the patient and family, and 
TABLE 2. Sample Discussion Items During a Sentinel Hospitalization

Patient/family understanding of disease process and treatment outcomes

Patient/family understanding of disease prognosis

Availability of alternative treatment options including palliative/hospice care

Patient/family wishes/goals of care

Advanced-care planning including limitations of care

Inventory of symptoms (frequency, severity, modifying factors, timing, and treatments)

Social and financial stress

Emotional and existential stress

Social support system and caregivers

Living arrangements

to encourage discussions about goals of care and advanced care planning during the sentinel hospitalization. It also provides a structured vehicle for soliciting the patient's (and family's) perspectives and documenting them in the medical record. A compilation of sample items to guide discussion can be found in Table 2 . Hospitalists, equipped with basic palliative care skills and supported by hospital- and community-based palliative care teams, can thrive in this unique position of optimizing the quality of care for these patients. ${ }^{40}$ Almost 20 years ago, the field of palliative care rose to national prominence on the findings of the SUPPORT (Study to Understand Prognoses and Preferences for Outcomes and Risks of Treatments) study, which investigated the suboptimal end-of-life experiences of hospitalized adult patients. ${ }^{41}$ Since then, the fields of both hospital medicine and palliative care have grown, yet the best is still to come for their collaborative excellence, mutual education, and shared care innovation at the forefront of medicine.

Disclosure: Nothing to report.

\section{References}

1. Chadaga SR, Maher MP, Maller N, Mancini D, Mascolo M, Sharma $\mathrm{S}$, et al. Evolving practice of hospital medicine and its impact on hospital throughput and efficiencies. J Hosp Med. 2012;7:649-654.

2. Hogan C, Lunney J, Gabel J, Lynn J. Medicare beneficiaries' costs of care in the last year of life. Health Affairs. 2001;20:188-195.

3. Meier DE. Palliative care in hospitals. J Hosp Med. 2006;1:21-28.

4. Pantilat SZ. Palliative care and hospitalists: a partnership for hope. $J$ Hosp Med. 2006;1:5-6.

5. Muir JC, Arnold RM. Palliative care and the hospitalist: an opportunity for cross-fertilization. Am J Med. 2001;111:10s-14s.

6. Swetz KM, Kamal AH. Palliative care. Ann Intern Med. 2012;156: ITC2-1, TC2-2-15; quiz TC2-16.

7. Lunney JR, Lynn J, Foley DJ, Lipson S, Guralnik JM. Patterns of functional decline at the end of life. JAMA. 2003;289:2387-2392.

8. Mitchell SL, Teno JM, Kiely DK, et al. The clinical course of advanced dementia. N Engl J Med. 2009;361:1529-1538.

9. Christakis NA, Lamont EB. Extent and determinants of error in doctor's prognoses in terminally ill patients: prospective cohort study. BMJ. 2000;320:469-472.

10. Moss AH, Lunney JR, Culp S, et al. Prognostic significance of the "surprise" question in cancer patients. J Palliat Med. 2010;13:837-840.

11. Moss AH, Ganjoo J, Sharma S, et al. Utility of the "surprise" question to identify dialysis patients with high mortality. Clin J Am Soc Nephrol. 2008;3:1379-1384.

12. Walter LC, Brand RJ, Counsell SR, et al. Development and validation of a prognostic index for 1-year mortality in older adults after hospitalization. JAMA. 2001;285:2987-2994.

13. Cowen ME, Strawderman RL, Czerwinski JL, Smith MJ, Halasyamani LK. Mortality predictions on admission as a context for organizing care activities. J Hosp Med. 2013,8:229-235.
14. Youngwerth J, Min S, Statland B, Allyn R, Fischer S. Caring about prognosis: a validation study of the CARING criteria to identify hospitalized patients at high risk for death at 1 year. J Hosp Med. 2013,8: 696-701.

15. Downing M, Lau F, Lesperance M, Karlson N, Shaw J, Kuziemsky C, et al. Meta-analysis of survival prediction with palliative performance scale. J Palliat Care. 2007;23:245-254.

16. Yourman LC, Lee SJ, Schonberg MA, Widera EW, Smith AK. Prognostic indices for older adults: a systemic review. JAMA. 2012;307: 182-192.

17. Weissman DE, Meier DE. Identifying patients in need of a palliative care assessment in the hospital setting. J Palliat Med. 2011;14:17-23.

18. Tamura MK, Covinsky KE, Chertow GM, Yaffe K, Landefeld CS, McCulloch CE. Functional status of elderly adults before and after initiation of dialysis. N Engl J Med. 2009;361:1539-1547.

19. Strand JJ, Kamdar MM, Carey EC. Top 10 things palliative care clinicians wished everyone knew about palliative care. Mayo Clin Proc. 2013;88:859-865.

20. Curtis JR. Palliative and end-of-life care for patients with severe COPD. Eur Respir J. 2008;32:796-803.

21. Goodlin SJ. Palliative care in congestive heart failure. J Am Coll Cardiol. 2009;54:386-396.

22. Quill TE, Abernethy AP. Generalist plus specialist palliative carecreating a more sustainable model. N Engl J Med. 2013;368:11731175 .

23. Cherlin E, Morris V, Morris J, Johnson-Hurzeler R, Sullivan GM, Bradley EH. Common myths about caring for patients with terminal illness: opportunities to improve care in the hospital setting. J Hosp Med. 2007;2:357-365.

24. Zaros MC, Curtis JR, Silveira MJ, Elmore JG. Opportunity lost: endof-life discussions in cancer patients who die in the hospital. J Hosp Med. 2013;8:334-340.

25. Case AA, Orrange SM, Weissman DE. Palliative medicine physician education in the United States: a historical review. J Palliat Med. 2013;16:230-236.

26. Widera E, Pantilat SZ. Hospitalization as an opportunity to integrate palliative care in heart failure management. Curr Opin Support Palliat Care. 2009;3:247-251.

27. Walling AM, Asch SM, Lorenz KA, et al. The quality of care provided to hospitalized patients at the end of life. Arch Intern Med. 2010;170: $1057-1063$.

28. Eti S, O'Mahony S, McHugh M, Guilbe R, Blank A, Selwyn P. Outcomes of the acute palliative care unit in an academic medical center [published online ahead of print May 10, 2013]. Am J Hosp Palliat Care. doi: 10.1177/1049909113489164.

29. Anderson WG, Flint LA, Horton JR, Johnson K, Mourad M, Sharpe BA. Update in hospital palliative care. J Hosp Med. 2013;12:715720.

30. Temel JS, Greer JA, Muzikansky A, et al. Early palliative care for patients with metastatic non-small-cell lung cancer. $N$ Engl J Med. 2010;363:733-742.

31. Greer JA, Jackson VA, Meier DE, Temel JS. Early integration of palliative care services with standard oncology care for patients with advanced cancer. CA Cancer J Clin. 2013;63:349-363.

32. Jack BW, Chetty VK, Anthony D, et al. A reengineered hospital discharge program to decrease rehospitalization: a randomized trial. Ann Intern Med. 2009;150:178-187.

33. Ahalt C, Walter LC, Yourman L, Eng C, Perez-Stable EJ, Smith AK. "Knowing is better": preferences of diverse older adults for discussion prognosis. J Gen Intern Med. 2012;27:568-575.

34. Bomba PA, Kemp M, Black JS. POLST, an improvement over traditional advanced directives. Cleve Clin J Med. 2012;79:457-464.

35. Ali AA, Adam R, Taylor D, Murchie P. Use of a structured palliative care summary in patients with established cancer is associated with reduced hospital admissions by out-of-hours general practitioners in Grampian [published online ahead of print January 3, 2013]. BMJ Support Palliat Care. doi:10.1136/bmjspcare-2012-000371.

36. Aragon K, Covinsky K, Miao Y, Boscardin WJ, Flint L, Smith AK. Use of the Medicare posthospitalization skilled nursing benefit in the last 6 months of life. Arch Intern Med. 2012;172:1573-1579.

37. Brumley R, Enguidanos S, Jamison P, et al. Increased satisfaction with care and lower costs: results of a randomized trial of in-home palliative care. J Am Geriatr Soc. 2007;55:993-1000.

38. Enguidanos S, Vesper E, Lorenz K. 30-day readmissions among seriously ill older adults. J Palliat Med. 2012;15:1-6.

39. Hansen LO, Greenwald JL, Budnitz T, et al. Project BOOST: effectiveness of a multihospital effort to reduce rehospitalization. J Hosp Med. 2013;8:421-427.

40. Kutner JS. Ensuring safe, quality care for hospitalized people with advanced illness, a core obligation for hospitalists. J Hosp Med. 2007; 2:355-356

41. The SUPPORT Principal Investigators. A controlled trial to improve care for seriously ill hospitalized patients: the Study to Understand Prognoses and Preferences for Outcomes and Risks of Treatments (SUPPORT). JAMA. 1995;274:1591-1598. 